\title{
Shaping Couzin-like Torus Swarms through Coordinated Mediation
}

\author{
Shin-Young Jung, Daniel S. Brown, and Michael A. Goodrich \\ Computer Science \\ Brigham Young University \\ Provo, Utah, USA \\ ldsrogan@byu.edu
}

\begin{abstract}
Human-swarm interaction methods often allow a human to influence a swarm through either leadership or predation. These methods of influence have two main limitations: (1) although leaders sustain influence over nominal agents for a long period of time, they tend to cause all collective structures to turn in to flocks (negating the benefit of other swarm formations) and (2) predators tend to cause collective structures to fragment We introduce the use of mediators as a novel shared control method for human-swarm influence and use mediators to shape Couzin-like tori [1]. The mediator method uses special agents that operate from within the spatial center of a swarm. This approach allows a human operator to transform and move a dynamic torus formation while sustaining influence over the torus, avoiding fragmentation, and maintaining the torus' connectivity. The use of mediators allows a human to mold and adapt the torus' behavior and structure to a wide range of spatio-temporal tasks such as military protection and decontamination tasks.

Index Terms-multi-agent systems, swarm control, shared control
\end{abstract}

\section{INTRODUCTION}

A swarm consists of a group of simple individuals who exhibit collective behaviors. In swarms, each individual moves without input from a centralized controller, responding spontaneously to signals from its environment and its neighbors.

A handful of recent flocking and swarming algorithms use three simple heuristic rules [1]-[3]: (1) each individual attempts to stay within a certain range of its neighbors, (2) each individual tries to avoid collisions by maintaining a minimum distance from its neighbors, and (3) each individual matches its velocity to its neighbors. Because these simple rules can produce a range of mobile spatial structures, which we call Couzin-like structures, swarms that follow these rules can potentially be applied to many domains such as military force protection, firefighting, search and rescue, etc. [4], [5].

A swarm that is formed by only these simple rules has limited communication, and, consequently, it is non-trivial to shape and guide the way these structures move. One way to allow a swarm to achieve complex goals and adapt to changing environments is to increase the complexity and sophistication of the individual agents. Another way, that minimizes the complexity of individual agents, is to enable a human to influence the swarm, but share control over the swarm's behavior with the individual agents. Enabling human interaction allows swarm algorithms to be flexible in solving complex problems and doing other meaningful tasks such as transporting and collecting objects.

Human influence on swarm intelligence can be categorized as one of four primitive human-swarm interactions [6]. These primitive interactions fall into two different categories of human influence over a swarm: direct and indirect communication [6]. The first way a human can influence a swarm directly is by changing agent parameters such as velocity, turning rate, and the zone of influence. For example, changing parameters can cause agents to display different patterns of movement [1], [3]. The second direct way is to use persistent influence. This method requires one or more operators who are aware of the current situation and can influence the swarm by giving continuous input [2], [5]. The third direct way is through intermittent interactions. This can be done by having the human input goals that the swarm needs to achieve. By using these direct communication methods, a human can control many aspects of swarm behaviors. By contrast, a human can also influence a swarm's behavior indirectly by changing features of the environment. This can be accomplished, for example, by an operator who sets up a beacon in the environment to influence how a swarm will move [7], [8].

We introduce a novel way to control a swarm by applying persistent influence via direct communication with agents called mediators. We explore two types of mediators. One mediator type repels agents similar to the way a predator does [9], [10]. We show that $(a)$ setting the parameters of individual agents allows these agents to maintain a certain distance from the predator-based mediator and $(b)$ the agents can be made to exhibit encircling motions around the mediators. The other mediator type has the same influence zones as previous leader models [9]-[11], but also includes a repulsion region within the attraction region. This mediator type allows a human to alter the collective shape exhibited by agents as they encircle a group of mediators. We provide details about these models later in this paper.

Existing swarm models are typically capable of either flocking [2], [3], [12] or torus behavior [13], [14], and in some cases can exhibit multiple group behaviors depending on the model parameters used [1], [15]. Flocking is effective for 
quickly moving a cohesive group of agents to a new location. Individuals in a flock can monitor only the front area because all agents head toward the same direction. Conversely, the torus is effective for performing stationary tasks or creating a perimeter. Because agents in a torus move in circular trajectories they provide omnidirectional sensing. This paper focuses on controlling a torus using mediators so that the swarm can travel and change shape while staying cohesive and not changing individual agents' parameters.

\section{RELATED WORK}

Previous work on Human Swarm Interaction (HSI) has described several different methods for controlling the agents in a swarm. Kira and Potter used virtual leaders to influence a swarm [12]. Similar to their control method, Olfati-Saber also used a virtual leader to control the behavior of a flock [2]. Su et al. proposed a method for controlling a flock using multiple virtual leaders where agents have limited sensing capabilities [16]. Mabrouk et al. use a virtual leader to escape from a local minima in a reactive problem domain [17]. While these papers deal with enabling human interaction with swarms through virtual leaders, our approach adds human interaction to a swarm through one or more physical agents that attract and repel agents to shape and steer a torus.

Prior work on exerting human influence over swarms by either leadership or predation has illustrated two limitations: (1) although leaders sustain influence over nominal agents for a long period of time, they tend to cause all collective structures to turn in to flocks (essentially negating the potential benefit of the torus formation) [9]; (2) predators tend to cause collective structures to fragment, creating a series of small flocks or small tori [9]. We propose a class of mediator agents that allow a human to sustain influence over a torus, change its shape, and move the torus while keeping it intact.

Elkaim and Kelbley showed formation shapes that are similar to those that we propose. They used virtual leaders with attraction potential forces [18]. The basic concept of their approach is to maintain an equilibrium between interagent potential forces and the forces applied by a virtual leader positioned at the centroid of the agents. The difference between their model and our model is that our mediator model uses both repelling and attracting forces and keeps these forces constant, whereas their leader agents influence other agents using only a scaled attraction force.

Varghese and McKee manipulate agent position by calculating a geometric transformation that makes each agent move to the right position while avoiding collisions with obstacles [19]. Kawashima et al. investigate the responsiveness of fixed-communication leader-follower networks for manipulating multi-agent formations [20]. Our model does not require calculating a geometric transformation or fixed communication topology, but instead, agents move based on three simple rules and are able to form various formations through the influence of mediators.

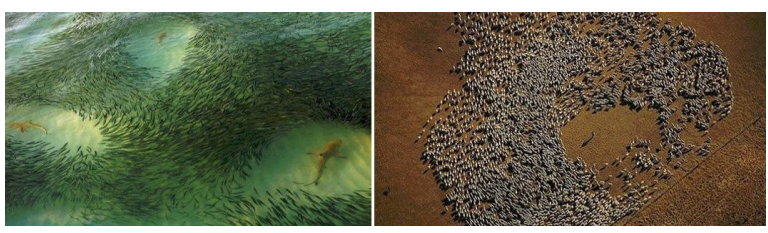

Fig. 1. Predators with agents in nature.

\section{THE MODEL}

The model we propose is biomimetic, meaning biologically inspired [1]. Fig. 1 shows how agents in nature respond to either their neighbors or to predators, producing round empty space around the predators. Based on this natural behavior, we propose two different agent types: nominal agents and mediators. Mediators are directly influenced by the human, but the nominal agents are influenced indirectly via mediators. This means that the human and nominal agents share control over the specific structure of the swarm because the human can influence nominal agents only by appropriately managing mediators.

We adopt a switchingbased control model in which the nominal agents either $(a)$ react to their neighbors or $(b)$ react to the mediators; see Fig. 2. This gives shared control between a human and a swarm. The human provides input to the mediators, and

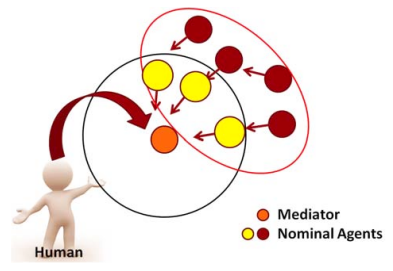

Fig. 2. Switching-based control model. the mediators influence nominal agents that are within range of the mediators. Since the nominal agents may move in and out of the sensing range of the mediator, agents can switch back and forth between inter-agent influence and mediator influence. Because avoiding collisions is critical, we also added a switch in which nominal agents ignore mediators if interagent distance drops below a threshold.

\section{A. Nominal Agent}

The nominal agent uses a two-dimensional implementation of Couzin's three dimensional model [1]. Since we are interested in ground robots, the two-dimensional model is sufficient. As mentioned previously, this model uses three basic rules and can produce two fundamentally different structures: a torus and a flock [1], [3]. The first rule is that each agent attempts to stay close to other agents. This is accomplished by the zone of attraction $\left(R^{a t t}\right)$. Agents attract to neighbors within the zone of attraction to maintain swarm connectivity. The second rule is that each agent tries to avoid collisions with other agents by maintaining a minimum inter-agent distance. This is accomplished by the zone of repulsion $\left(R^{r e p}\right)$. This rule has the highest priority [1]. This means that an agent will ignore attraction and orientation forces in order to avoid a neighbor within its zone of repulsion. The third rule is that each agent matches its velocity and direction with its neighbors. This is accomplished by the zone of orientation $\left(R^{\text {ori }}\right)$. 


\section{B. Mediators}

In the previous section, we reviewed how nominal agents determine their behavior through inter-agent zones of repulsion, orientation, and attraction. We also indicated that these nominal agents change their behavior when they are in proximity to a mediator, ignoring all inter-agent influences except repulsion and responding only to the mediator.

This means that nominal agents need two sets of parameters for determining their actions: a set of parameters for when they are in the presence of a mediator, and a set of parameters for when they are not in the presence of a mediator. It is useful to treat the former set as a property of the mediator rather than the nominal agent. This allows us to systematically explore how nominal agents respond to different types of mediators.

Mediators exert two forms of influence over the nominal agents: leadership and predation. For the purpose of this paper, leadership means the mediators exert attractive influence over nominal agents, pulling nominal agents toward them. Conversely, predation means the mediators exert a repelling influence over nominal agents, pushing nominal agents away from themselves.

The first type of mediator, which we call an R-mediator for repulsion mediator, influences nominal agents using only predation, but uses what we can call a "weak" form of predation. Weak predation means the mediator repels nominal agents, but the zone of mediator predation is smaller than the zone of nominal attraction. Let the zone of predation be denoted by $R^{\text {pred }}$. Weak predation occurs when $2 \times R^{\text {pred }}<R^{\text {att }}$, or equivalently $R^{\text {pred }}<R^{\text {att }} / 2$, which means that the range of influence between nominal agents exceeds the maximum range of $\mathrm{R}$-mediator influence on the nominal agents. This allows nominal agents to stay in a cohesive torus formation when a mediator is in the center of the group. Combining this constraint with parameters that Couzin used to produce a torus yields the following ordering of parameters:

$$
R^{\text {rep }} \leq R^{\text {ori }}<R^{\text {pred }}<R^{\text {att }} / 2 .
$$

This allows a mediator to be in the middle of a torus and "steer" the torus in various directions, as shown in Fig. 3.

Note that this means that the nominal agents use the attraction, orientation, and repulsion behaviors identified in the previous section when not in the presence of a mediator; when a mediator is nearby, the mediator repels the agents and the agents ignore each other except when avoiding collisions.

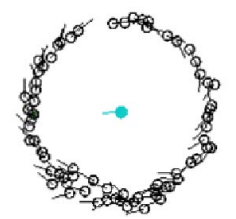

There are some limitations to this form of mediator-based influence. First, it is difficult to place the mediator into the center of a swarm once the structure is broken. Additionally, if there are not enough nominal agents, the torus behavior of a swarm around the mediator becomes fragmented because the nominal agents don't have enough attractive influence from other nominal agents to stay within a certain range of the mediator.
To overcome these limitations, we introduce an alternative mediator, called an RA-mediator for repulsion and attraction mediator. The RA-mediator uses a zone of leadership wherein the mediator attracts the nominal agents. As mentioned above, when a leader agent uses attraction only, it tends to cause all collective structures to turn into flocks. To avoid this, we require the RA-mediator to include both attraction, corresponding to a zone of leadership denoted by $R^{\text {lead }}$, and repulsion, corresponding to a zone of predation denoted by $R^{\text {pred }}$.

Because mediators operate from within the "hole" of the torus, we note that the following parameter ordering allows the mediator to control the behavior of the torus:

$$
R^{\text {rep }} \leq R^{\text {ori }}<R^{\text {pred }}<R^{\text {att }}<R^{\text {lead }} .
$$

Setting $R^{\text {pred }}<R^{\text {att }}<R^{\text {lead }}$ creates a buffer zone around the mediator, allowing agents to stay close to the mediator but not too close.

Table I shows how the parameters of the R- and RAmediators relate to previous work using leaders and predators [9]. The first two rows in the table indicate the parameters used in prior models, and the last two rows indicate parameters for

\begin{tabular}{|c|c|c|}
\hline \multicolumn{2}{|c|}{ Influencer } & Order of Nominal Agent's Each Zone \\
\hline \multicolumn{2}{|c|}{ Leader } & $R^{r e p} \leq R^{\text {ori }}<R^{\text {att }}<R^{\text {lead }}$ \\
\hline \multicolumn{2}{|c|}{ Predator } & $R^{\text {rep }} \leq R^{\text {ori }}<R^{\text {att }}<R^{\text {pred }}$ \\
\hline \multirow{2}{*}{ Mediator } & $\mathrm{R}$ & $R^{\text {rep }} \leq R^{\text {ori }}<R^{\text {pred }}<R^{\text {att }} / 2$ \\
\hline & RA & $R^{\text {rep }} \leq R^{\text {ori }}<R^{\text {pred }}<R^{\text {att }}<R^{\text {lead }}$ \\
\hline
\end{tabular}
the two types of mediators introduced in this paper.

TABLE I

HOW NOMINAL AGENTS ARE INFLUENCED. TOP TWO INFLUENCERS INDICATE PREVIOUS MODELS.

Using these two different models for mediators, we simulated different forms of human interaction and analyzed the sensitivity of each model to human influence. Before we present our simulation results, we first derive a theoretical result for the maximum speed of a moving torus formation in terms of the nominal agent speed.

\section{Maximum Speed of a Torus}

We can think of the torus formation as a rotating disc of radius $r$. If we consider one agent moving along the perimeter of the torus and if we assume the torus is itself moving (i.e. the centroid of the torus has a certain velocity) then we can think of an individual agent's trajectory as the cycloid shown in Fig. 4. The parametric equations that gov-

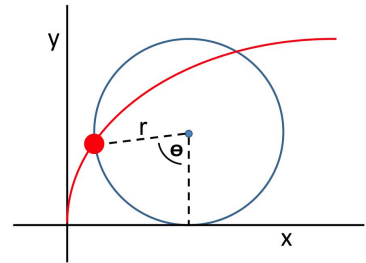

Fig. 4. Trajectory of an agent represented as a cycloid generated by a circle of radius $r$ that has rotated through the angle $\theta$. ern the motion of a cycloid generated by a circle of radius $r$ and parameterized by $\theta$, the angle through which the rolling circle has rotated, are $x=r(\theta-\sin \theta), y=r(1-\cos \theta)$. To 
determine the distance that the agent travels we can calculate the length of the parametric curve, denoted by $L$, where

$$
L=\int_{a}^{b} \sqrt{\left(\frac{d x}{d \theta}\right)^{2}+\left(\frac{d y}{d \theta}\right)^{2}} d \theta .
$$

So, the length of one arch of the cycloid is

$$
L=\int_{0}^{2 \pi} \sqrt{(r(1-\cos \theta))^{2}+(r \sin \theta)^{2}} d \theta=8 r .
$$

Assuming that the agent has a speed of $s$ units per second, we want to find the speed of the centroid of the torus, $s_{\text {torus }}$. The time taken for the agent to traverse the arc length is $8 \mathrm{r} / \mathrm{s}$. The center of the disc, or centroid of the torus, has traveled $2 \pi r$ units, therefore

$$
\max \left(s_{\text {torus }}\right)=\frac{2 \pi r}{8 r / s}=\frac{\pi}{4} s .
$$

Thus, the upper limit on the speed of a torus is approximately three-fourths the speed of an individual agent. Applying this theoretical result to the notion of nominal agents and mediators provides us with a way to calculate the maximum speed of a mediator given a fixed nominal agent speed. If the mediator moves faster than this maximum speed, the nominal agents will not be able to stay in a cohesive torus formation. Alternatively, given a fixed mediator speed, we can calculate the minimum nominal agent speed required to keep the torus cohesive.

\section{Sensitiviy Analysis}

We ran a series of experiments to analyze the sensitivity and robustness of human-swarm interactions through the use of mediators. We investigated different combinations of parameter settings to determine conditions for stable swarm formations. The parameters for a nominal agent are the ranges of zones of attraction $\left(R^{\text {att }}\right)$, repulsion $\left(R^{r e p}\right)$, orientation $\left(R^{\text {ori }}\right)$, as well as speed $(s)$, turning rate $(\omega)$, and vision range $(\theta)$. The parameters for mediators are the range of predation $\left(R^{\text {pred }}\right)$, the range of mediator-attraction $\left(R^{\text {lead }}\right)$, and speed $\left(s^{m}\right)$.

We measured distances in terms of units where $R^{r e p}$ is fixed as 1 unit because it is the minimum distance that is required to avoid collisions. Furthermore, we adopted Couzin's model parameters for vision range $(\theta)$ and turning rate $(\omega)$ (namely, $\theta=270^{\circ}$ and $\omega=40^{\circ} / \mathrm{sec}$ ) to ensure that the nominal agents exhibit the same collective behaviors as Couzin's model when not under the influence of a mediator. We also set the number of agents $(N)=70$ and $R^{\text {pred }}=14$. The other parameters for both nominal and mediator agents were varied.

In the following experiments, the goal is for the mediator to guide the torus through a series of four waypoints, returning to the starting location at the end of the circuit. Each waypoint is each vertex of $80 \times 80$ unit square. The objective is to find parameter ranges that afford stable human-influenced movement of a torus through mediators.

To determine whether the torus remained stable during simulations, we checked two conditions: nominal-connectivity and mediator-controllability. Nominal-connectivity is a condition that allows us to determine whether nominal agents are connected to each other. Mediator-controllability is a condition on the distance from the swarm-centroid to the mediator, allowing us to determine whether the mediator is in the center of the swarm and can effectively influence the agents.

The nominal-connectivity condition is $\sum_{k=1}^{N} A_{i j}^{k} \neq 0 \forall i, j$ where $A$ is the $N \times N$ adjacency matrix, $N$ is the number of agents, and $\mathrm{i}$ and $\mathrm{j}$ are agent indices. The centroid of a swarm $C$ is calculated as $C=\frac{1}{N} \sum_{i=0}^{N} P_{i}$ where $P_{i}$ is position of agent $i$. The distance from the swarm-centroid to the mediator is dist $^{m c}=\left\|P_{m}-C\right\|$ where $P_{m}$ is the position of the mediator. The mediator-controllable condition is $d i s t^{m c}<R^{\text {pred }}$.

Simulation results show that $R^{\text {att }}, R^{\text {ori }}$, and $R^{\text {lead }}$ do not have much effect on the average distance from the mediator to the swarm's centroid. We also varied the speed of the mediator, $s^{m}$, and the speed of the nominal agents, $s$, and found that these also have little effect on the average difference between the swarm-centroid and the position of the mediator.

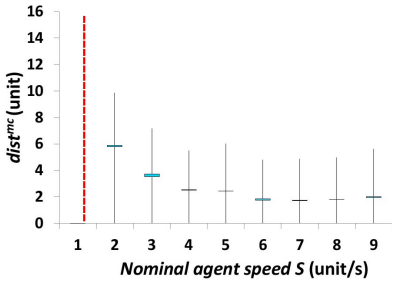

(a) Mediator speed $s^{m}=1$ unit $/ \mathrm{s}$

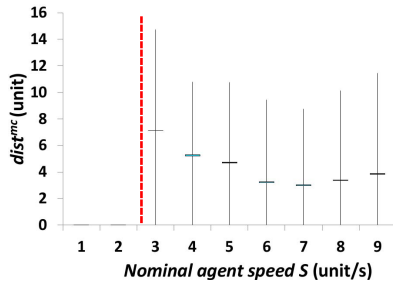

(b) Mediator speed $s^{m}=2$ unit $/ \mathrm{s}$
Fig. 5. Distance from the swarm-centroid to an R-mediator as a function of nominal agents' speed. Each line indicates the minimum and maximum distance from the swarm-centroid to the R-mediator. The upper bound of the box is the mean, and the lower bound of the box is the median. The dashedline is the minimum speed of the nominal agents, derived from Eq. 4. The results for an RA-mediator were similar.

However, as shown in Fig. 5, as the speed of a nominal agent is increased, dist ${ }^{m c}$ tends to decrease and then stay relatively constant. We also observed that the position of the nominal agents tended to be lopsided if the speed of the nominal agents is not fast enough to follow the mediator.

To further investigate this phenomenon, we kept track of the number of nominal agents on the left and right side of a moving mediator. In these simulations, the mediator starts moving to the right. After about 801 time steps, the mediator turns around and moves to the left. We fixed the speed of mediator to $s^{m}=1$ and varied the speed of the nominal agents. As shown in Fig. 6, if nominal agents move faster, their positions are almost evenly distributed around the mediator. By contrast, if the nominal agents move slower, their positions are lopsided to either the left or right side of the mediator.

These simulation results show that given a certain mediator speed, if the nominal agents move faster, the mediator will be more likely to stay in the middle of the torus. These results agree with the theoretical results of Section IV where we showed that the speed of the individual agents must be sufficiently faster than the speed of the centroid.

Based on the foregoing experiments, we were able to find suitable ranges of parameters for the nominal agent that afforded robust human influence via a single mediator (see Table II). Note that the parameters are constrained based on Table I. We also observed that the suitable speed of a mediator 


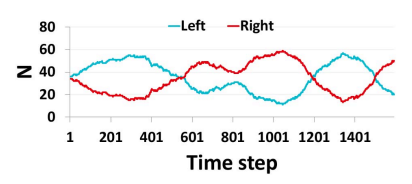

(a) $s=2$ unit $/ \mathrm{s}$

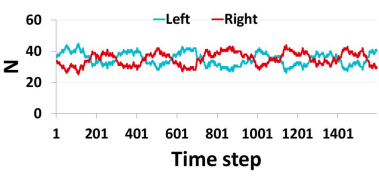

(c) $s=4 \mathrm{unit} / \mathrm{s}$

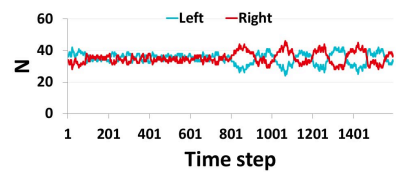

(e) $s=6$ unit $/ \mathrm{s}$

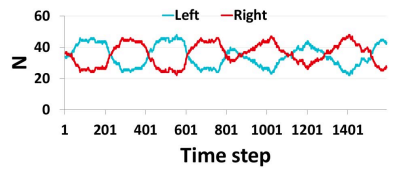

(b) $s=3 \mathrm{unit} / \mathrm{s}$

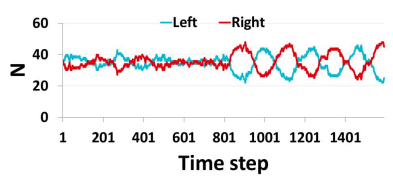

(d) $s=5$ unit $/ \mathrm{s}$

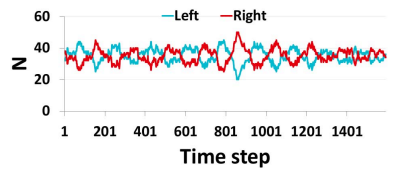

(f) $s=7$ unit $/ \mathrm{s}$
Fig. 6. These graphs display the change of the number of nominal agents at a mediator's right and left side. After around 801 time steps, the number of nominal agents at each side fluctuates because the mediator changes its direction to left from right.

\begin{tabular}{|c||c|c|c|}
\hline \multicolumn{1}{|c||}{} & \multicolumn{3}{c|}{ Parameters } \\
\hline Influencer & $R^{\text {ori }}$ & $R^{\text {att }}$ & $R^{\text {lead }}$ \\
\hline \hline R-Mediator & $1-7$ & $31-35$ & - \\
\hline RA-Mediator & $1-7$ & $23-30$ & $25-35$ \\
\hline
\end{tabular}

TABLE II

SUMMARY OF NOMINAL AGENTS PARAMETER BASED ON THEIR INFLUENCER.

$s^{m}$ is restricted by the speed of a nominal agent $s$. As shown in Fig. 5 and Section IV, torus behavior around a mediator appears when $s^{m} \leq \frac{\pi}{4} s$. When $s^{m} \geq 3$, we need to increase the turning rate for the nominal agents to prevent the torus from fragmenting. Thus, the suitable speed of the mediator is 0-2 units/s when $\omega$ is restricted to $40^{\circ} / \mathrm{sec}$.

\section{SHAPING SWARMS}

If we place more than one mediator in the center of a swarm, we can make the nominal agents track many different perimeter shapes. If multiple mediators are given a specific initial configuration and move with the same direction and the same speed, the shape of the swarm is approximately static as the group translates to a new location. Fig. 7 shows how a group of mediators can manipulate the shape of a swarm. Also, the shape of a swarm can be changed dynamically by the human operator by moving the mediators. For example, the bar shape can be transformed into triangle or many other desired shapes.
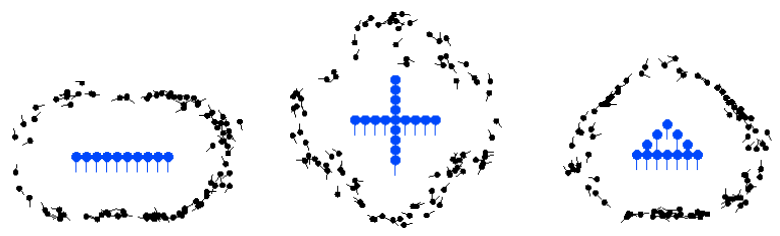

Fig. 7. Tori Shapes.
To extend this idea further, we introduce a new type of nominal agent, which we call the smart agent. Smart agents, or S-agents, are inspired by the behavior of the sheep illustrated in Fig. 8. In this figure, the sheep are orbiting a moving car. Because the car covers part of the sheep's vision, the sheep cannot see the entire group's movement. Rather, they can see only the neighbors in front of them so they follow those neighbors.

Likewise, if S-agent $i$ in a swarm observes a set of neighbors $O$, it decides to follow the closest neighbor $E_{i}$ where

$$
E_{i}=\underset{j \in O}{\operatorname{argmin}}\left(\sqrt{\left(i_{x}-j_{x}\right)^{2}+\left(i_{y}-j_{y}\right)^{2}}\right) .
$$

This corresponds to a nearest neighbor topology which has been shown to accurately model interactions in natural flocks [21]. The main difference between an S-agent and a nominal agent is that an $\mathrm{S}$-agent has a more narrow field of view $\left(\theta=180^{\circ}\right.$ rather than $\left.270^{\circ}\right)$. Because an S-agent has a larger blind spot, it needs more than just attraction to maintain the connectivity of the swarm.

In order to make the agents "smarter", each agent $i$ remembers the last location of its closest neighbor, $P_{E_{i}}$. When an S-agent does not observe any neighbors within its vision, the S-agent recalls the last location of its closest neighbor and moves towards that location.

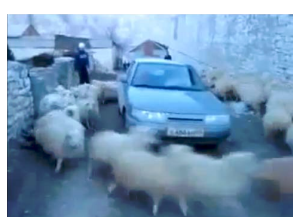

Fig. 8. Sheep's encircling motion around a car.
As soon as the S-agent observes a neighbor, it responds to the observed neighbor and resets its memory.

Another way of being smarter so as to maintain connectivity is that each S-agent has the ability to increase its speed when it gets far from its nearest neighbor [22]. The speed for agent $i$ is

$$
s_{i}(t+1)= \begin{cases}\gamma \times s & \text { if }\left\|P_{i}-P_{E_{i}}\right\|>\text { Stable Dist } \\ s & \text { otherwise }\end{cases}
$$

where $s$ is constant and $\gamma>1$ determines how much the agent increases its speed. Using the same simulation setup as in Section V, we found that when Stable Dist $\leq 0.9 \times R^{\text {att }}$ and $\gamma \geq 1.1$, the torus remained stable during the simulations. Also, we found that the mediator needs to move slower with $\mathrm{S}$-agents than with nominal agents to maintain a stable torus formation.

Fig. 9 shows the different topologies that result when using mediators with either nominal agents or S-agents. Nominal agents show more influence dependencies than S-agents. This means that nominal agents respond to more neighbors than $\mathrm{S}$-agents do in order to maintain their connectivity-S-agents need only the closest neighbor in front of them. Consequently, using S-agents slides the weight of shared control from being highly weighted on nominal agents to being equally weighted between mediators and S-agents. Because of this change, Sagents are better suited to shaping swarms than nominal agents. 

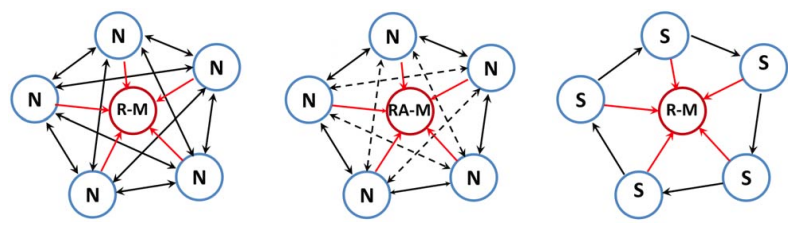

Fig. 9. Topologies among nominal agents $N$, S-agents $S$, and mediators $R$ and $R A-M$. Black lines indicate the response among nominal agents. Dashedlines indicate that nominal agents may respond to each other depending on the range of influence. Red lines indicate the response to the mediator. The notation $a \rightarrow b$ means $a$ is influenced by $b$.

Fig. 10 illustrates that a group of S-agents under the influence of a group of R-mediators can adopt a set of very flexible shapes, more than is possible with nominal agents under the influence of a group of mediators. This preliminary observation is encouraging for two reasons. First, unlike prior work on predator-based or leader-based influence, mediators allow us to manage agents in a torus shape. Second, we can "warp and bend" the shape of the torus by a proper positioning of mediators.

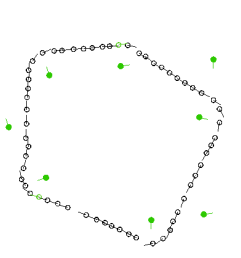

(a)

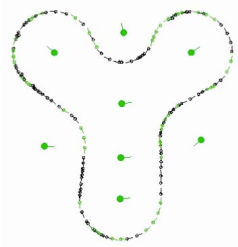

(b)
Fig. 10. Dynamic transformation of S-agents from an amorphous blob (a) to a $\mathrm{Y}$ shape (b) under the influence of a group of coordinating R-mediators.

\section{CONCLUSION AND FUTURE WORK}

We introduced a new shared control model for humanswarm interaction using mediators and demonstrated that this model can be used to transform a swarm into a variety of shapes. Because mediator-based swarm control allows a swarm to maintain a torus formation while it is moving, the swarm retains the advantages of torus behavior, in contrast to previous work on leader and predator based control. We also analyzed the sensitivity of this model and found that there is a wide range of parameters for both nominal agents and mediators that allow a mediator to stay in the middle of a stable moving torus. In future work, we will study whether mediated swarms can be robustly applied in the noisy conditions which will exist with real robots. Because individual agents in our model use constant speed and limited turning rates-which is similar to Dubins path algorithm commonly used for robot path planning [23] - we hope to apply our mediator model to real robots in the future. Future work will also examine how robustly the mediator model can handle a variety of shapes.

\section{ACKNOWLEDGMENT}

We would like to thank the Science of Autonomy program of the Office of Naval Research for funding this work. The opinions of this paper do not necessarily reflect the funding agency.

\section{REFERENCES}

[1] I. Couzin, J. Krause, R. James, G. Ruxton, and N. Franks, "Collective memory and spatial sorting in animal groups," J. of Theoretical Biology, vol. 218 , no. 1 , pp. 1-11, 2002.

[2] R. Olfati-Saber, "Flocking for multi-agent dynamic systems: Algorithms and theory," IEEE Trans on Automatic Control, vol. 51, no. 3, pp. 401420, 2006.

[3] C. Reynolds, "Flocks, herds and schools: A distributed behavioral model," in ACM SIGGRAPH Computer Graphics, vol. 21, no. 4. ACM, 1987, pp. 25-34.

[4] R. Wiegand, M. Potter, D. Sofge, and W. Spears, "A generalized graphbased method for engineering swarm solutions to multiagent problems," Parallel Problem Solving from Nature-PPSN IX, pp. 741-750, 2006.

[5] L. Alboul, J. Saez-Pons, and J. Penders, "Mixed human-robot team navigation in the guardians project," in IEEE Intl. Wkshop. on Safety, Security and Rescue Robotics, 2008. IEEE, 2008, pp. 95-101.

[6] M. Lewis, personal communication, 2012.

[7] A. Kolling, S. Nunnally, and M. Lewis, "Towards human control of robot swarms," in Proceedings of the seventh annual ACM/IEEE intl. conf. on Human-Robot Interaction. ACM, 2012, pp. 89-96.

[8] J. Werfel and R. Nagpal, "Extended stigmergy in collective construction," Intelligent Systems, IEEE, vol. 21, no. 2, pp. 20-28, 2006.

[9] A. Goodrich, P. Sujit, S. Kerman, B. Pendleton, and J. Pinto, "Enabling human interaction with bio-inspired robot teams: Topologies, leaders, predators, and stakeholders." Technical Report BYU-HCMI Technical Report 2011-1, Brigham Young University, Tech. Rep., 2011.

[10] A. Wood and G. Ackland, "Evolving the selfish herd: emergence of distinct aggregating strategies in an individual-based model," Proc. of the Royal Society B: Biological Sciences, vol. 274, no. 1618, pp. 16371642, 2007.

[11] I. Couzin, J. Krause, N. Franks, and S. Levin, "Effective leadership and decision-making in animal groups on the move," Nature, vol. 433, no. 7025, pp. 513-516, 2005.

[12] Z. Kira and M. Potter, "Exerting human control over decentralized robot swarms," in 4th Intl. Conf. on Autonomous Robots and Agents, 2009. IEEE, 2009, pp. 566-571.

[13] J. Marshall, M. Broucke, and B. Francis, "Formations of vehicles in cyclic pursuit," IEEE Trans. on Automatic Control, vol. 49, no. 11, pp. 1963-1974, 2004.

[14] H. Levine, W. Rappel, and I. Cohen, "Self-organization in systems of self-propelled particles," Physical Review E, vol. 63, no. 1, p. 017101, 2000 .

[15] D. Strömbom, "Collective motion from local attraction," J. of Theoretical Biology, vol. 283, no. 1, pp. 145-151, 2011.

[16] H. Su, X. Wang, H. Dong, and G. Chen, "Flocking with multiple virtual leaders based on position measurements," in 7th Asian Control Conf., 2009. IEEE, 2009, pp. 1156-1161.

[17] M. Mabrouk and C. McInnes, "Swarm robot social potential fields with internal agent dynamics," in 12th International Conference on Aerospace Sciences and Aviation Technology, ASAT-12, 2007.

[18] G. Elkaim and R. Kelbley, "A lightweight formation control methodology for a swarm of non-holonomic vehicles," in Aerospace Conf. IEEE, 2006, pp. 8-pp.

[19] B. Varghese and G. McKee, "Investigating feasible tools for swarm pattern transformation," in Second Intl. Conf. on Robot Communication and Coordination, 2009. IEEE, 2009, pp. 1-7.

[20] H. Kawashima, G. Zhu, J. Hu, and M. Egerstedt, "Responsiveness and manipulability of formations of multi-robot networks," in 2012 IEEE 51st Annual Conference on Decision and Control. IEEE, 2012, pp. 4622-4628.

[21] M. Ballerini, N. Cabibbo, R. Candelier, A. Cavagna, E. Cisbani, I. Giardina, V. Lecomte, A. Orlandi, G. Parisi, A. Procaccini et al., "Interaction ruling animal collective behavior depends on topological rather than metric distance: Evidence from a field study," Proc. of the National Academy of Sciences, vol. 105, no. 4, p. 1232, 2008.

[22] N. Bode, D. Franks, and A. Wood, "Limited interactions in flocks: relating model simulations to empirical data," J. of The Royal Society Interface, vol. 8, no. 55, pp. 301-304, 2011.

[23] L. E. Dubins, "On curves of minimal length with a constraint on average curvature, and with prescribed initial and terminal positions and tangents," American J. of Mathematics, vol. 79, no. 3, pp. 497-516, 1957. 